\title{
Comunicación
}

\section{Crecimiento Pre-Destete del Ovino F1 Blackbelly x Pelibuey en Condiciones de Pastoreo Libre en la Amazonía Ecuatoriana}

\author{
Pre-Weaning Growth of F1 Blackbelly x Pelibuey Sheep under Free Grazing \\ Conditions in the Ecuadorian Amazon
}

\author{
Juan Carlos Moyano ${ }^{1,6,7}$, Juan Carlos López ${ }^{1,6}$, Pablo Roberto Marini ${ }^{2,3,6}$, \\ M.L. Fischman ${ }^{4,5,6}$
}

\section{Resumien}

El objetivo del trabajo fue describir las curvas de crecimiento del ovino F1 Blackbelly $x$ Pelibuey desde el nacimiento hasta el destete, en condiciones de pastoreo libre en la Amazonía ecuatoriana. Se trabajó con 12 machos y 10 hembras desde el nacimiento hasta el destete. Los animales fueron pesados al nacimiento, a los 4 y 9 días de edad y luego semanalmente hasta los 55 días de edad (destete). El desarrollo corporal fue lineal. No existieron diferencias significativas de peso al nacimiento, peso al destete y ganancia diaria de peso entre sexos.

Palabras clave: oveja; crecimiento; región amazónica

\section{Abstract}

The aim of this study was to describe the growth curves of F1 Blackbelly x Pelibuey sheep from birth to weaning under free grazing conditions in the Ecuadorian Amazon. Twelve males and 10 females were used in the study. The animals were weighed at birth,

\footnotetext{
${ }^{1}$ Centro de Investigación, Posgrado y Conservación Amazónica, Universidad Estatal Amazónica, Ecuador

${ }^{2}$ Facultad de Ciencias Veterinarias, Universidad Nacional de Rosario, Argentina

${ }^{3}$ Consejo de Investigaciones (CIC-UNR), Rosario, Argentina

${ }^{4}$ Laboratorio de Calidad Espermática y Criopreservación de Gametas, ${ }^{5}$ Instituto de Investigación y Tecnología en Reproducción Animal (INITRA), Facultad de Ciencias Veterinarias, Universidad de Buenos Aires, Argentina

${ }^{6}$ Centro Latinoamericano de Estudios de Problemáticas Lecheras (CLEPL), Casilda, Provincia de Santa Fe, Argentina

${ }^{7}$ E-mail: juancamt@hotmail.com
}

Recibido: 11 de abril de 2017

Aceptado para publicación: 25 de agosto de 2017 
at 4 and 9 days of age and then weekly until 55 days of age (weaning). Body development was linear. There were no significant differences in birth weight, weaning weight and daily weight gain between sexes.

Key words: sheep; growth; Amazon region

\section{INTRODUCCIÓN}

Las razas ovinas Blackbelly y Pelibuey son de gran relevancia en la producción de carne ovina en la Amazonía ecuatoriana. Estas explotaciones permiten un ingreso económico por la venta de los corderos, además de ser una alternativa productiva que permite explotar la zona sin afectar el bosque nativo. Ambas razas son rústicas y adaptadas al complicado medioambiente amazónico. La forma de explotación, en condiciones extensivas a pastoreo libre, constituye uno de los componentes de equilibro entre el ecosistema y las producciones familiares, lo que podría ser uno de los pilares de la sostenibilidad ambiental y social de la Amazonía.

En Ecuador, la explotación de la ganadería ovina es de tipo extensivo, y se desenvuelve bajo el sistema tradicional, con razas criollas y mestizas. Existen comunidades de indígenas que han utilizado razas especializadas (Corriedale, Ramboulliet, Cheviot, Poll Dorset) como inicio de un programa de mejoramiento genético realizado por el MAG hace 25 años y que actualmente es administrado por la Asociación Nacional de Criadores de Ovejas (ANCO). Bajo este sistema, se aprovecha las áreas de pastos naturales, principalmente en los páramos andinos, actividad que constituye el sustento familiar. En el último censo agropecuario se registraron 1127468 ovinos (Oñate, 2003).

En los años 90 se dio inicio a la ganadería de ovinos de pelo, y actualmente su población se puede estimar en 1000 animales, distribuidos entre 120-140 fincas con una media de 7 a 8 ovejas por finca. Las razas
Barbados Blackbelly (localmente llamados Barriga Negra) y Pelibuey-West African (localmente llamados Sudán) son las razas predominantes, cada una con $40 \%$ del total de la población, mientras que la diferencia corresponde a cruces con ovejas de lana (Claus et al., 2014).

La eficiencia productiva de este sistema de producción requiere ser evaluado dentro de un contexto ambiental y sociocultural específico. Para ello, se debería buscar la mejor productividad individual por oveja en función de los ingresos generados durante su ciclo productivo, los cuales dependen del número de corderos por parto, del peso y ganancias registradas por estos, de la longevidad de la oveja y de los ingresos obtenidos a la venta de los animales. Es así que debe mejorarse la capacidad de crecimiento de los corderos, la prolificidad, la longevidad productiva y la calidad de la carne y la canal (Lupi et al., 2015).

Este trabajo tuvo como objetivo describir las curvas de crecimiento del ovino Blackbelly x Pelibuey desde el nacimiento hasta el destete, en condiciones de pastoreo libre, en la Amazonía ecuatoriana.

\section{Materiales Y Métodos}

El estudio se realizó en el Centro de Investigación, Posgrado y Conservación de la Biodiversidad Amazónica (CIPCA), ubicado en el cantón Arosemena Tola, provincia de Napo (Ecuador). El ambiente es tropical con temperaturas que varían entre los 15 y $25^{\circ} \mathrm{C}$, precipitación pluvial anual de $4000 \mathrm{~mm}$, hu- 
Cuadro 1. Promedio y error estándar del peso al nacimiento, peso al destete y ganancia de peso entre el nacimiento y el destete de corderos F1 Blackbelly x Pelibuey en la región amazónica del Ecuador

\begin{tabular}{lccc}
\hline Sexo & $\begin{array}{c}\text { Peso al } \\
\text { nacimiento } \\
(\mathrm{kg})\end{array}$ & $\begin{array}{c}\text { Peso al destete } \\
(\mathrm{kg})\end{array}$ & $\begin{array}{c}\text { Ganancia de peso } \\
\text { por día } \\
(\mathrm{kg})\end{array}$ \\
\hline Machos $(\mathrm{n}=12)$ & $2.9 \pm 0.2^{\mathrm{a}}$ & $11.5 \pm 1^{\mathrm{a}}$ & $0.126 \pm 0.03^{\mathrm{a}}$ \\
Hembras $(\mathrm{n}=10)$ & $2.7 \pm 0.2^{\mathrm{a}}$ & $10.6 \pm 1^{\mathrm{a}}$ & $0.116 \pm 0.02^{\mathrm{a}}$ \\
\hline
\end{tabular}

medad relativa del $80 \%$, con topografía caracterizada por relieves ligeramente ondulados sin pendientes pronunciadas, distribuidos en mesetas naturales de gran extensión. La altitud varía entre los 580 y $990 \mathrm{msnm}$. Si bien los suelos presentan una composición muy heterogénea, la mayoría se origina en sedimentos fluviales procedentes de la región andina del país.

La institución dispone de 42 ha de pastos destinada a la lechería. Se trabajó con los 22 ovinos cruzados Blackbelly x Pelibuey (F1) que disponía el CIPCA. Estos son 12 machos y 10 hembras, que se les hizo el seguimiento desde el nacimiento hasta el destete. Los animales fueron pesados al nacimiento y a los 4 y 9 días y luego cada semana hasta los 65 días de edad en que se destetaron. Los pesos se obtuvieron en el mismo horario y con una balanza digital Etekcity con capacidad para $50 \mathrm{~kg}$.

El peso de las madres Blackbelly entre el parto y el destete fue de $37.6 \pm 6.8 \mathrm{~kg}$. Todas estuvieron entre el primer y tercer parto y tuvieron partos simples. Todos los animales fueron criados bajo las mismas condiciones ambientales, nutricionales y de manejo. Se utilizó un solo macho Pelibuey para todas las hembras.

Los animales se mantuvieron en pastoreo libre, sobre pasturas con áreas específicas de Brachiaria decumbens, $B$. brizantha, Arachis pintoi, Desmodium ovalifolium y Stylosanthes guianensis. Se aplicó el manejo sanitario habitualmente empleado para el rodeo ovino del CIPCA, que incluye desparasitaciones, baños contra garrapatas y moscas, y vacunaciones contra fiebre aftosa, rabia y estomatitis vesicular.

Se estimaron los promedios y errores estándar para las variables peso al nacimiento, peso al destete y aumento de peso diario. Se determinó la posible diferencia estadística entre sexos mediante el análisis de varianza a un criterio de clasificación y las medias se compararon con la prueba de Turkey-Kramer $(p<0.05)$, utilizando el programa JMP v. 5.0 para Windows.

\section{Resultados}

En el Cuadro 1 se observan los pesos al nacimiento y al destete, así como la ganancia diaria de peso en los animales en estudio. Se observó una tendencia no significativa de un mejor desarrollo corporal de los machos sobre las hembras.

Los cambios de peso corporal de los corderos tuvieron un mejor ajuste con una regresión lineal, tanto en machos $\left(\mathrm{R}^{2}=0.84\right)$ como en hembras $\left(\mathrm{R}^{2}=0.85\right)$. En ambos casos se observa una ligera declinación en el crecimiento a partir de los 51 días de edad (Figura 1). 

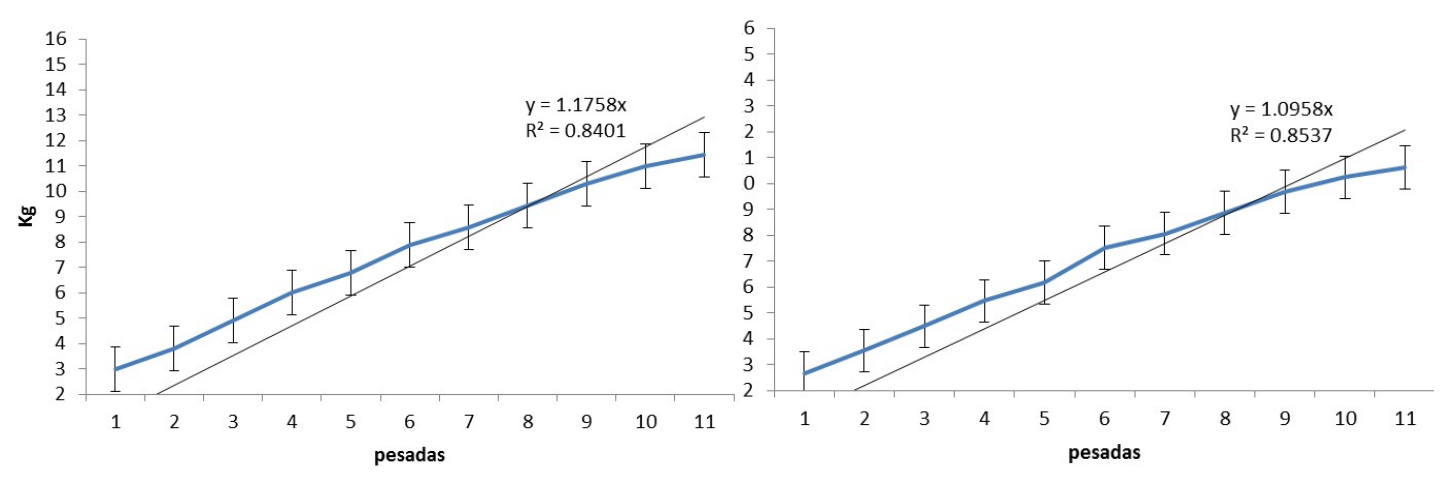

Figura 1. Evolución del peso de corderos F1 Blackbelly x Pelibuey machos (izquierda) y hembras (derecha) en la región amazónica del Ecuador

\section{Discusión}

Los corderos, como en la mayoría de los mamíferos en la etapa de crecimiento predestete, dependen fundamentalmente de la leche de la madre. En el ovino, el pico de mayor producción de leche coincide con la etapa de crecimiento más acelerado de los corderos, hasta los primeros 41 días de edad (Méndez y Shimada, 1982). Esto mismo se observa en el presente trabajo, donde el crecimiento más acelerado ocurre hasta el día 51 de edad.

Las ganancias de peso fueron menores a las reportadas para corderos Blackbelly suplementados con una ración con $14 \%$ de proteína (Cabrera et al., 1996) y para corderos Pelibuey x Blackbelly suplementados con una ración con $20 \%$ de proteína (Moguel y Acuña, 1989).

Existen otros factores que afectan el peso de los corderos al nacimiento y al destete como son el tipo y número de parto, el sexo, la época del año y el manejo de la madre (Carrillo et al., 1987). El sexo y el número de corderos nacidos por parto son dos de los factores que ejercen mayor influencia sobre el crecimiento de los ovinos (De Lucas et al., 2003). A medida que aumenta el nú- mero de crías nacidas por parto disminuye el peso al nacimiento (Robinson et al., 1977; Rodríguez et al., 1998; Quesada et al., 2002); en forma similar, los corderos provenientes de partos simples presentan una mayor tasa de crecimiento pre y posdestete con respecto a aquellos provenientes de partos con me1lizos (Dimsoski et al., 1999; González et al., 2002).

No se encontraron diferencias de pesos al nacimiento ni al destete entre sexos en el presente estudio. Las ganancias diarias de peso (machos: $126 \mathrm{~g}$; hembras: $116 \mathrm{~g}$ ) fueron menores a los valores de 160, 144 y $139 \mathrm{~g}$ reportados en corderos de varias razas de pelo (Hinojosa-Cuéllar et al., 2009), Pelibuey (Hinojosa-Cuéllar et al., 2012) y en cruces de razas de pelo con razas de lana, pero similares a los $122 \mathrm{~g}$ encontrados en corderos Blackbelly (González et al., 2002). Sin embargo, otros trabajos realizados en varias razas y bajo diferentes sistemas de producción, tampoco indicaron diferencias atribuidas al sexo para el peso al nacimiento de los corderos (Quintero et al., 1997) ni para el crecimiento predestete (González et al., 2002). Incluso, se dispone de estudios que señalan mayores tasas de crecimiento a los 30 y 60 días para las hembras (Gbangboche et al., 2006). 


\section{Conclusión}

Los corderos F1 Blackbelly x Pelibuey presentan un ritmo de crecimiento lineal entre el nacimiento y el destete, sin diferencia significativa entre sexos.

\section{Literatura Citada}

1. Cabrera TE, Montes PR, Delgado de LR, Castellanos RA. 1996. Efecto de la suplementación fosforada en pre y posparto a ovejas Pelibuey y sobre el comportamiento de las crías. Mem Reunión Nacional de Investigación Pecuaria en México. Morelos, México.

2. Carrillo L, Velásquez A, Ornelas T. 1987. Algunos factores ambientales que afectan el peso al nacer y al destete de corderos Pelibuey. Téc Pec Méx 25: 289295. doi: 10.22319/rmcp.v25i3.3474

3. Claus C, Fischer J, Herrera A, Rahmann G. 2014. La ganadería de ovinos de pelo para un uso sustentable en la zona periférica de bosque tropical de América del Sur. Deutsche Gesellschaft für Technische Zusammenarbeit (GTZ), Asistencia Técnica Alemana Tropenökologisches Begleitprogramm (TÖB) y Programa de Apoyo Ecológico. Universidad de Kassel. 80 p.

4. De Lucas TJ, Zarco LA, González E, Tórtora E, Villa-Godoy A, Vázquez C. 2003. Crecimiento predestete de corderos en sistemas intensivos de pastoreo y manejo reproductivo en el altiplano central de México. Vet Méx 34: 235-245.

5. Dimsoski P, Tosh J, Clay JC, Irvin KM. 1999. Influence of management system on litter size, lamb growth, and carcass characteristics in sheep. J Anim Sci 77: 1037-1043.

6. Gbangboche AB, Youssao AK, Senou M, Adamou-Ndiaye M, Ahissou A, Farnir F, Michaux C. 2006. Examination of non-genetic factors affecting the growth performance of Djallonke sheep in Soudanian zone at the Okpara breeding farm of Benin. Trop Anim Health Prod 38: 55-64.

7. González, R, Torres G, Castillo M. 2002. Crecimiento de corderos Blackbelly entre el nacimiento y el peso final en el trópico húmedo de México. Vet Méx 33: 443-453.

8. Hinojosa-Cuéllar JA, RegaladoArrazola FM, Oliva-Hernández J. 2009. Crecimiento prenatal y predestete en corderos Pelibuey, Dorper, Katahdin y sus cruces en el sureste de México. Rev Cient FCV-LUZ 19: 522-532.

9. Hinojosa-Cuéllar JA, Oliva-Hernández J, Torres-Hernández G, SeguraCorrea JC, Aranda-Ibáñez EM, González-Camacho JM. 2012. Factores que afectan el crecimiento predestete de corderos Pelibuey en el trópico húmedo de México. Univ Cienc 28: 163171.

10. Lupi TM, Nogales S, León JM, Delgado JV. 2015. Modelación de curvas de crecimiento comercial en ovino Segureño. AICA 6: 132-143.

11. Méndez CD, Shimada SA. 1982. Requerimientos nutritivos del cordero lactante de la raza Tabasco. Mem XV Reunión Anual de Investigaciones Pecuarias. México.

12. Moguel CFJ, Acuña AMA. 1989. Comportamiento de corderos Pelibuey $\mathrm{x}$ Blackbelly alimentados con harina de yuca a diferentes edades del destete. Mem Reunión Nacional de Investigación Pecuaria. México DF.

13. Oñate RH. 2003. Informe sobre recursos zoogenéticos Ecuador: Ministerio de Agricultura y Ganadería. $30 \mathrm{p}$.

14. Quintero A, Boscán J, Palomares $R$, González A, Boissiere J. 1997. Efecto del sexo sobre el peso corporal a diferentes edades en corderos West-African criados en el trópico venezolano. Arch Latinoam Prod Anim 5 (Supl 1): 426- 427.

15. Quesada M, McManus C, D'Araújo Couto FA. 2002. Efeitos genéticos e fenotípicos sobre caracteristicas de 
produção e reprodução de ovinos deslanados no Distrito Federal. Rev Bras Zootec 31: 342-349. doi: 10.1590/S151635982002000200008

16. Robinson JJ, McDonald I, Fraser C, Crafts RMJ. 1977. Studies in reproduction in prolific ewes. I. Growth of the products of conception. J Agric Sci 88: 539-552.

17. Rodríguez OL, Heredia M, Quintal J, Velázquez A. 1998. Productivity of Pelibuey and Blackbelly ewes mated at yearly and 8-monthly intervals over six years. Small Ruminant Res 30: 177-184. 